$17^{\text {th }}$ European Symposium on Computer Aided Process Engineering - ESCAPE17

V. Plesu and P.S. Agachi (Editors)

(C) 2007 Elsevier B.V./Ltd. All rights reserved.

\title{
A Tool for Kalman Filter Tuning
}

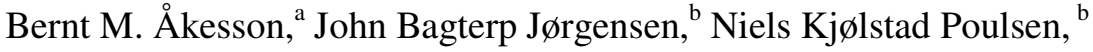 \\ Sten Bay Jørgensen ${ }^{\mathrm{a}}$ \\ ${ }^{a}$ CAPEC, Department of Chemical Engineering, Technical University of Denmark, \\ 2800 Lyngby, Denmark, baa@kt.dtu.dk, sbj@kt.dtu.dk \\ ${ }^{b}$ Informatics and Mathematical Modelling, Technical University of Denmark, \\ 2800 Lyngby,Denmark, jbj@imm.dtu.dk, , nkp@imm.dtu.dk
}

\begin{abstract}
The Kalman filter requires knowledge about the noise statistics. In practical applications, however, the noise covariances are generally not known. A method for estimating noise covariances from process data has been investigated. The method gives a least-squares estimate of the noise covariances, which can be used to compute the Kalman filter gain.
\end{abstract}

\section{Keywords}

Kalman filter; Covariance estimation; State estimation

\section{Introduction}

In state estimation the state of a system is reconstructed from process measurements. State estimation has important applications in control, monitoring and fault detection of chemical processes. The Kalman filter and its counterpart for nonlinear systems, the extended Kalman filter, are wellestablished techniques for state estimation. However, a well-known drawback of Kalman filters is that knowledge about process and measurement noise statistics is required from the user. In practical applications the noise covariances are generally not known. Tuning the filter, i.e. choosing the values of the process and measurement noise covariances such that the filter performance is optimized with respect to some performance index, is a 
challenging task. If performed manually in an ad hoc fashion it represents a considerable burden for the user. Therefore there is need for a tool that can perform filter tuning or provide assistance to the user.

The filter tuning problem is essentially a covariance estimation problem and the Kalman filter gain is computed based on the estimated covariances. This issue has been addressed in numerous papers and a number of methods have been presented, cf. discussion in [1,2] and references therein. A promising technique for covariance estimation is the autocovariance least-squares method proposed recently by Odelson and co-workers for linear time-invariant systems [1]. This method is based on the estimated autocovariance of the output innovations, which is used to compute a least-squares estimate of the noise covariance matrices. The estimation problem can be stated in the form of a linear leastsquares problem with additional constraints to ensure positive semidefiniteness of the covariance matrices.

In this paper, a generalized autocovariance least-squares tuning method is applied to the Kalman filter. This Kalman filter tuning methodology is implemented into a software tool to facilitate practical applications.

The performance of the Kalman filter tuning tool (Kalfilt) is demonstrated on a numerical example.

\section{Problem statement}

Consider a linear time-invariant system in discrete-time,

$$
\begin{aligned}
x_{k+1} & =A x_{k}+B u_{k}+G w_{k} \\
y_{k} & =C x_{k}+v_{k}
\end{aligned}
$$

where $A \in \mathrm{R}^{n \times n}, B \in \mathrm{R}^{n \times m}, G \in \mathrm{R}^{x \times g}$ and $C \in \mathrm{R}^{p \times n}$. The process noise $w_{k}$ and the measurement noise $v_{k}$ are zero-mean white noise processes with covariance matrices $Q_{w}$ and $R_{v}$, respectively, and cross-covariance $S_{w v}$. Assume that a stationary Kalman filter is used to estimate the state. The one-step ahead prediction is given by

$$
\hat{x}_{k+1 k}=A \hat{x}_{k \mid k-1}+B u_{k}+K_{p}\left(y_{k}-C \hat{x}_{k \mid k-1}\right)
$$

where the Kalman filter gain $K_{p}$ is defined as 


\section{A Tool for Kalman Filter Tuning}

$$
K_{p}=\left(A P_{p} C^{T}+G S_{w v}\right)\left(C P_{p} C^{T}+R_{v}\right)^{-1}
$$

and $P_{p}$ is the covariance of the state prediction error, $\tilde{x}_{k \mid k-1}=x_{k}-\hat{x}_{k \mid k-1}$. The

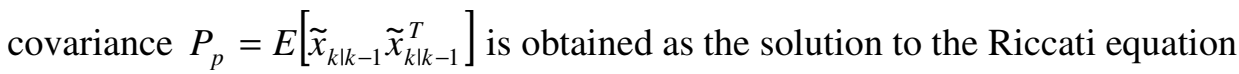

$$
\begin{aligned}
P_{p} & =A P_{p} A^{T}+G Q_{w} G^{T} \\
& -\left(A P_{p} C^{T}+G S_{w v}\right)\left(C P_{p} C^{T}+R_{v}\right)^{-1}\left(C P_{p} A^{T}+S_{w v}^{T} G^{T}\right)
\end{aligned}
$$

In the following approach it is assumed that the model is given, along with an initial suboptimal Kalman filter, based on initial guesses $Q_{w, 0}, R_{v, 0}$ and $S_{w v, 0}$. The objective is to estimate the covariance matrices $Q_{w}, R_{v}$ and $S_{w v}$ and use these to compute the Kalman filter gain $K_{p}$.

\section{Paper approach}

\subsection{Methodology}

A general state-space model of the measurement prediction error can be defined,

$$
\begin{aligned}
\tilde{x}_{k+1 \mid k} & =\left(A-K_{p} C\right) \tilde{x}_{k \mid k-1}+G w_{k}-K_{p} v_{k} \\
e_{k} & =C \tilde{x}_{k \mid k-1}+v_{k}
\end{aligned}
$$

where $e_{k}=y_{k}-C \hat{x}_{k \mid k-1}$.

The autocovariance of the measurement prediction or estimate error is given by

$$
\begin{aligned}
R_{e, 0} & =E\left[e_{k} e_{k}^{T}\right]=C P_{p} C^{T}+R_{v} \\
R_{e, j} & =E\left[e_{k+j} e_{k}^{T}\right]=C\left(A-K_{p} C\right)^{j} P_{p} C^{T}+C\left(A-K_{p} C\right)^{j-1} G S_{w v} \\
& -C\left(A-K_{p} C\right)^{j-1} K_{p} R_{v}, \quad j \geq 1
\end{aligned}
$$


The autocovariance matrix is defined as

$$
R_{e}(L)=\left[\begin{array}{cccc}
R_{e, 0} & R_{e, 1}^{T} & \cdots & R_{e, L-1}^{T} \\
R_{e, 1} & R_{e, 0} & \cdots & R_{e, L-2}^{T} \\
\vdots & \vdots & \ddots & \vdots \\
R_{e, L-1} & R_{e, L-2} & \cdots & R_{e, 0}
\end{array}\right]
$$

Substitution of Eqs. (6) into Eq. (7) followed by separation of the right-hand side into terms is performed. After this, the vec operator is applied to both sides of the resulting equation. The vec operator performs stacking of the matrix columns to form a column matrix [3]. This allows the problem to be stated as a linear least-squares problem,

$$
\operatorname{vec}\left(R_{e}(L)\right)=A_{l s} \operatorname{vec}(\underbrace{\left[\begin{array}{cc}
Q_{w} & S_{w v} \\
S_{w v}^{T} & R_{v}
\end{array}\right]}_{X})
$$

where the parameter matrix $A_{l s}$ is formed from system matrices $A, G, C$ and the Kalman filter gain $K_{p}$. This has the form of a linear least-squares problem. The left-hand side of Eq. (8) can be estimated from steady-state data. Given a sequence of data $\left\{e_{i}\right\}_{i=1}^{N_{d}}$, the estimate of the autocovariance can be computed by

$$
\hat{R}_{e, j}=\frac{1}{N_{d}-j} \sum_{i=1}^{N_{d}-j} e_{i+j} e_{i}^{T}
$$

where $N_{d}$ is the length of the data sequence. An estimated autocovariance matrix $\hat{R}_{e}(L)$ can then be formed analogously to Eq. (7). Solving (8) as a linear leastsquares problem does not gurantee that the estimated covariance matrices are positive semidefinite. Furthermore, the parameter matrix $A_{l s}$ may be poorly conditioned, which affects the accuracy of the solution. This can be remedied by adding a regularization term. The estimation problem can be formulated as follows, 
$\min _{X}\{\underbrace{\left\|A_{l s} \operatorname{vec}(X)-\operatorname{vec}\left(\hat{R}_{e}(L)\right)\right\|_{2}^{2}}_{\Phi}+\lambda \underbrace{\left\|\operatorname{vec}\left(X-X_{0}\right)\right\|_{2}^{2}}_{\Phi_{r}}\}$

s.t. $X$ symmetric positive semidefinite.

where $\lambda$ is a regularization parameter chosen by the user and allows a suitable bias-variance trade-off. Eq. (10) is a semidefinite least-squares problem, which is convex and can be solved by an interior point method [1]. A suitable value for $\lambda$ can be found by plotting $\Phi_{r}$ versus $\Phi$ for different values of $\lambda$. The optimal Kalman filter gain can then be computed from the estimated covariances using Eq. (3) after solving the Riccati equation (4).

\subsection{Numerical example}

We consider a system with the following system matrices

$$
A=\left[\begin{array}{ccc}
0.1 & 0 & 0.1 \\
0 & 0.2 & 0 \\
0 & 0 & 0.3
\end{array}\right], \quad G=\left[\begin{array}{l}
1 \\
2 \\
3
\end{array}\right], \quad C=\left[\begin{array}{lll}
0.1 & 0.2 & 0
\end{array}\right]
$$

and noise covariances $Q_{w}=0.5, R_{v}=0.1$ and $S_{w v}=0.2$. A set of 200 simulations were performed, each comprising a sequence of $N_{d}=1000$ data points. For each simulation, the covariances were estimated. The autocovariance lag was chosen as $L=15$.

The effect of regularization was investigated for the first simulation. In Fig. 1 the regularization term $\Phi_{r}$ and the fit to data $\Phi$ are plotted versus each other for parameter values $\lambda \in\left[10^{-9}-10\right]$. The parameter value $\lambda=0.1$ gave a good trade-off $\left(\Phi_{r}=0.15\right)$ and was used subsequently. The covariance estimates are plotted in Fig. 2. The bias in the estimates is due to regularization.

The performance of the tuned Kalman filter is compared to that of the initial filter and the ideal filter based on perfect information. The root-mean square error of output predictions, 
$R M S E P=\sqrt{\frac{1}{N_{d}} \sum_{k=1}^{N_{d}}\left\|y_{k}-C \hat{x}_{k \mid k-1}\right\|_{2}^{2}}$

is computed for each experiment and the result is shown in Fig. 3. As can be seen, the prediction error of the tuned filter is very close to that of the ideal filter, despite the bias in the estimates.

\section{Conclusions}

A tool for Kalman filter tuning was presented. The method used is a generalization of the autocovariance least-squares method to systems with mutually correlated noise. Regularization was included in order to handle illconditioning of the least-squares problem. The results show that significant improvement to the predictions can be achieved can be achieved with the Kalman filter tuning tool.

\section{Acknowledgements}

The authors would like to thank Murali Rajamani and Professors James B. Rawlings and Per Christian Hansen for their help.

\section{References}

[1] B.J. Odelson, M.R. Rajamani and J.B. Rawlings, Automatica 42 (2006) 303.

[2] B.J. Odelson, A. Lutz and J.B. Rawlings, IEEE Trans. Contr. Syst. Technol. 14 (2006) 532.

[3] J.W. Brewer, IEEE Trans. Circuits Syst. 25 (1978) 772.

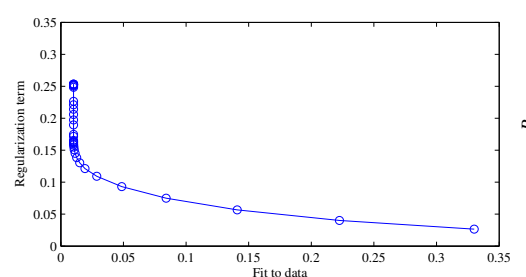

Figure 1: Trade-off curve.

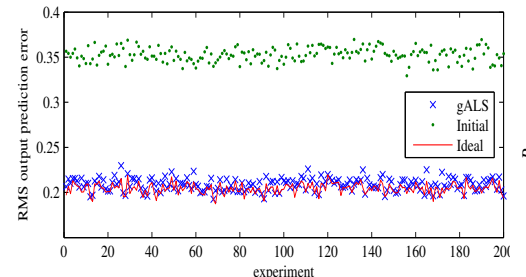

Figure 3: Prediction results.
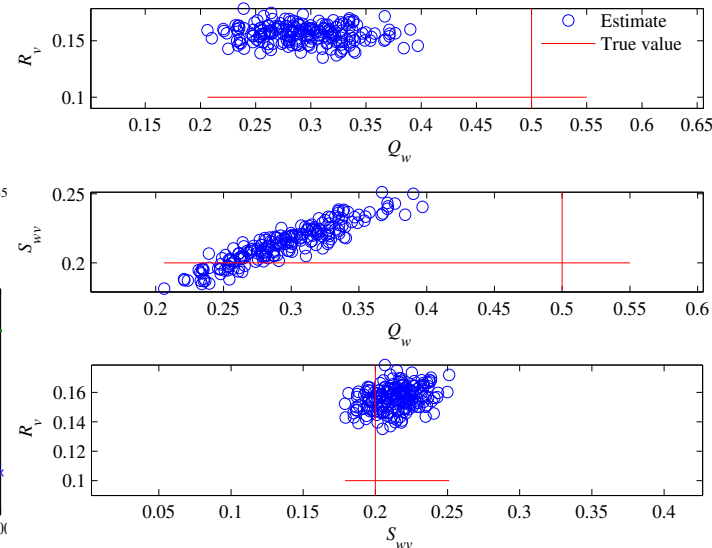

Figure 2: Estimated covariances. 